\title{
Levels of matrix metalloproteinase-9 and tissue inhibitor of metalloproteinase-1 are related to cardio- pulmonary injury in fetal inflammatory response syndrome
}

\author{
Yiwei Yan (D), Lian Jiang iD,* Mei Li iD, Huifen Zhang iD, Ying Shen iD, Wenhao Zhang iD, Wenting Zhang iD \\ Department of Pediatrics, The Fourth Hospital of Hebei Medical University, Shijiazhuang, China. \\ Yan Y, Jiang L, Li M, Zhang H, Shen Y, Zhang W, et al. Levels of matrix metalloproteinase-9 and tissue inhibitor of metalloproteinase-1 are related to \\ cardiopulmonary injury in fetal inflammatory response syndrome. Clinics. 2020;75:e2049 \\ *Corresponding author. E-mail: jianglianerke@163.com
}

\begin{abstract}
OBJECTIVES: To evaluate the diagnostic value of matrix metalloproteinase-9 (MMP-9), tissue inhibitor of metalloproteinase-1 (TIMP-1), and the MMP-9/TIMP-1 ratio in fetal inflammatory response syndrome (FIRS), and determine a possible association with the incidence of bronchopulmonary dysplasia (BPD) and myocardial injury. METHODS: Overall, 61 cases of preterm infants with FIRS were divided into the FIRS group 1 ( $\leqslant 32$ weeks) and FIRS group 2 (32 to 37 weeks). Similarly, 57 cases of normal preterm infants were divided into Control group 1 and Control group 2. Levels of interleukin-6 (IL-6), MMP-9, and TIMP-1 were detected by enzyme-linked immunosorbent assay. Spearman's linear correlation was used to analyze the relationship between dependent variables. Pathological changes were examined by hematoxylin and eosin (HE) staining and in amniotic fluid smears.
\end{abstract}

RESULTS: Levels of IL-6, MMP-9, and TIMP-1, and the MMP-9/TIMP-1 ratio were significantly higher in the FIRS group than in the Control groups. IL-6 was positively correlated with MMP-9, TIMP-1, and the MMP-9/TIMP-1 ratio. Areas under the curve (AUC) of MMP-9, TIMP-1, and the MMP-9/TIMP-1 ratio were 0.92, 0.90, and 0.95, respectively. HE staining and amniotic fluid smears showed the aggregation of inflammatory cells. MMP-9, TIMP-1, and the MMP-9/TIMP-1 ratio were closely related to the incidence of BPD ( $\leqslant 32$ weeks) and myocardial injury ( $<37$ weeks) in preterm infants.

CONCLUSION: MMP-9, TIMP-1, and the MMP-9/TIMP-1 ratio revealed a certain diagnostic value for FIRS; combined with gestational age, these parameters were effective for predicting cardiopulmonary injury.

KEYWORDS: Preterm Infants; Fetal Inflammatory Response Syndrome; Bronchopulmonary Dysplasia; Myocardial Injury.

\section{INTRODUCTION}

Preterm birth is characterized by birth at $<37$ weeks of gestation and generally causes neonatal morbidity and death (1). According to the reported births in 2018, the birth rate of premature infants increased from $9.93 \%$ to $10.02 \%$ (2). Notably, intrauterine infections are the most common cause of preterm infant births, accounting for approximately $40 \%$ of the overall cases of preterm births (3). Furthermore, intrauterine infections may cause the immune system to

Copyright $\odot 2020$ CLINICS - This is an Open Access article distributed under the terms of the Creative Commons License (http://creativecommons.org/licenses/by/ 4.0/) which permits unrestricted use, distribution, and reproduction in any medium or format, provided the original work is properly cited.

No potential conflict of interest was reported.

Received for publication on May 20, 2020. Accepted for publication on August 26, 2020

DOI: $10.6061 /$ clinics/2020/e2049 release numerous inflammatory factors, defined as the fetal inflammatory response syndrome (FIRS).

FIRS is characterized by a plasma interleukin-6 (IL-6) level $>11 \mathrm{pg} / \mathrm{mL}$ (4). Fortunately, fetal blood plasma can be obtained either by cordocentesis or from the umbilical blood plasma. Therefore, it provides an effective method for the early diagnosis of FIRS. Additional evidence has demonstrated that matrix metalloproteinases (MMPs) play important roles in pathological processes, including inflammation, cardiovascular diseases, and pulmonary diseases $(5,6)$. However, its activity is controlled by tissue inhibitors of metalloproteinases (TIMPs). As an important member of the MMP family, MMP-9 is a major component of the basement membrane of airways (7). Therefore, the correlation between IL-6 and MMP-9, TIMP-1, and the MMP-9/TIMP-1 ratio, as well as its diagnostic value in FIRS, warrant further exploration.

FIRS corresponds to the systemic inflammatory response syndrome (SIRS) observed in adult conditions, inducing the injuries to numerous organs during the neonatal period (8). Numerous studies have increasingly focused on injuries in the fetal lung, brain, and heart $(9,10)$ as imaging can be 
easily performed. As previously documented, bronchopulmonary dysplasia (BPD) and myocardial injury are common disorders in preterm infants $(11,12)$. Previous studies have indicated that IL- 6 and the MMP-9/TIMP-1 ratio are related to the pathogenesis of $\operatorname{BPD}(13,14)$. However, whether the MMP-9/TIMP-1 ratio is related to the pathogenesis of myocardial injury remains unclear.

The aim of this study was to evaluate the correlation between IL-6 and MMP-9, and TIMP-1, and the MMP-9/ TIMP-1 ratio in the umbilical cord blood of patients with FIRS. The findings of this study may help provide a new basis for predicting health disorders in preterm newborns.

\section{MATERIAL AND METHODS}

\section{Patients}

In total, 118 preterm infants born at $27^{+6}-36^{+5}$ weeks of gestation were selected from May 2014 to December 2015 at the Fourth Hospital of the Hebei Medical University. Overall, 61 infants who conformed to the diagnostic criteria of FIRS were included in the FIRS group, with the remaining infants (57 infants) included in the control groups. Among them, 33 cases in the FIRS group showed a gestational age $\leqslant 32$ weeks (FIRS group 1) and 28 cases showed a gestational age between 32-37 weeks (FIRS group 2). Similarly, 25 cases in the control group showed a gestational age of $\leqslant 32$ weeks (Control group 1) and 32 cases showed a gestational age between 32-37 weeks (Control group 2).

For FIRS, the main diagnostic indicators consisted of an IL6 level $>11 \mathrm{pg} / \mathrm{mL}$ in the umbilical cord blood, C-reactive protein (CRP) level $>6 \mathrm{mg} / \mathrm{L}$ in the amniotic fluid, inflammatory infiltration in the placenta and umbilical cord, and accumulation of inflammatory cells in the amniotic fluid. Other indicators were used as auxiliary diagnoses, including maternal fever, heart rate $>100$ beats per minute, fetal heart rate $>160$ beats per minute, uterine tenderness, amniotic fluid odor, and a peripheral blood white blood cell count $>1.5 \times 10^{10} / \mathrm{L}$. During follow-up, cases of BPD and myocardial injury were recorded separately, and the incidence of each was calculated. The protocol was approved by the Institutional Ethics Committee of the Fourth Hospital of Hebei Medical University (2020004), and informed consent was obtained from the parents of the infants.

\section{Sample collection}

Blood samples $(2-3 \mathrm{~mL})$ were obtained from the umbilical vein immediately after delivery and then centrifuged at $3000 \mathrm{rpm}$ for $10 \mathrm{~min}$. The serum was collected and stored at $-80^{\circ} \mathrm{C}$. Amniotic fluid samples $(2-3 \mathrm{~mL})$ were obtained during delivery. After mixing, amniotic fluid samples (0.2$0.3 \mathrm{~mL}$ ) were used to perform the smear test. The amniotic fluid samples were centrifuged at $3500 \mathrm{rpm}$ for $15 \mathrm{~min}$. Then, the supernatants were collected and stored at $-80^{\circ} \mathrm{C}$ until analysis. Once the placenta was delivered, tissues such as the placenta, umbilical cord, and fetal membranes were collected under relatively sterile conditions, placed in a neutral formaldehyde solution, and stored at $4^{\circ} \mathrm{C}$ until analysis.

\section{Enzyme-linked immunosorbent assay (ELISA)}

The serum concentrations of IL-6, MMP-9, and TIMP-1 were determined using double-antibody sandwich ELISA kits (Shanghai ELISA Biotech Co., Ltd., Shanghai, China), and the absorbance of the sample was measured using a microplate reader (Molecular Devices, VersaMax, California, USA).
All procedures were performed according to the manufacturer's instructions.

\section{Hematoxylin-eosin (HE) staining}

HE staining was performed to analyze pathological changes in the placenta. Briefly, tissues were sliced into $4-\mu \mathrm{m}$-thick sections, deparaffinized, and stained with HE. Images were captured with a microscope (Olympus CX-21, Olympus Corp., Tokyo, Japan).

\section{Examination of amniotic fluid smears}

In brief, amniotic fluid $(0.2-0.3 \mathrm{~mL})$ was placed dropwise onto a glass slide, and a uniform thin layer was formed. Then, Wright's stain (Wenzhou Kangtai Biotechnology Company, Wenzhou, China) was added, along with distilled water, in a dropwise manner. After mixing, the smear was washed and examined microscopically.

\section{Follow-up}

Data on hospitalization and post-discharge were collected, and preterm infants were followed up for 28 days. BPD and myocardial injury cases in the later period were recorded, and the incidence of BPD and myocardial injury was calculated.

For BPD, the diagnostic criteria were preterm infants who underwent mechanical ventilation or were treated with oxygen therapy and were still dependent on oxygen 28 days after birth. For myocardial injury, the diagnostic criteria were as follows: 1) elevated levels of myocardial enzymes; 2) elevated troponin levels; 3) abnormal electrocardiograph, including bradycardia and arrhythmia; 4) echocardiographic abnormalities, including altered heart structure, impaired systolic function, and impaired left ventricular ejection fraction.

\section{Statistical analysis}

Statistical analyses were performed using SPSS 13.0 (IBM Corp., Chicago, USA). The quantitative data are presented as the means \pm standard deviations (SDs) and were analyzed by Student's $t$-test for the comparison of data from two groups. Categorical data were analyzed using the $\chi^{2}$ test and expressed as a percentage. Spearman's linear correlation was used to analyze the relationship between dependent variables. Differences with $p$-values $<0.05$ were considered statistically significant.

\section{RESULTS}

\section{IL-6, MMP-9, and TIMP-1 were upregulated in the cord blood of preterm infants}

The clinical characteristics of the two groups are shown in Table 1 . There were no obvious differences in gestational age, sex, birth weight, and Apgar scores at $1 \mathrm{~min}, 5 \mathrm{~min}$, and 10 min between the FIRS and Control groups (all $p>0.05$ ). Furthermore, the current study revealed that the levels of IL6, MMP-9, and TIMP-1, and the MMP-9/TIMP-1 ratio in the FIRS group 1 with the same gestational age significantly increased compared with those in Control group 1 (all $p<0.01$ ). Additionally, the levels of IL-6, MMP-9, and TIMP1 , and the MMP-9/TIMP-1 ratio were higher in the FIRS group 2 than those observed in Control group 2 (all $p<0.01$ ) (Table 2).

Among preterm infants with FIRS, the levels of IL-6, MMP-9, and TIMP-1, and the MMP-9/TIMP-1 ratios were not statistically different between FIRS group 1 and FIRS 
Table 1 - General characteristics of the two groups.

\begin{tabular}{|c|c|c|c|c|}
\hline & FIRS group $(n=61)$ & Control group $(n=57)$ & t/F value & $p$-value \\
\hline Male/Female (n) & $32 / 29$ & $30 / 27$ & 0.00 & 0.99 \\
\hline Gestational age (weeks) & $29.35 \pm 1.44$ & $29.29 \pm 1.51$ & 0.22 & 0.83 \\
\hline Birth weight (g) & $1489 \pm 178$ & $1482 \pm 169$ & 0.22 & 0.83 \\
\hline Apgar score at $1 \mathrm{~min}$ & $7.28 \pm 1.42$ & $7.32 \pm 1.34$ & 0.16 & 0.88 \\
\hline Apgar score at $5 \mathrm{~min}$ & $8.39 \pm 1.52$ & $8.43 \pm 1.45$ & 0.15 & 0.88 \\
\hline Apgar score at $10 \mathrm{~min}$ & $9.01 \pm 1.26$ & $9.12 \pm 1.32$ & 0.46 & 0.64 \\
\hline
\end{tabular}

FIRS, Fetal inflammatory response syndrome.

Table 2 - Levels of IL-6, MMP-9, and TIMP-1, and the MMP-9/TIMP-1 ratio in the different groups.

\begin{tabular}{lcccc}
\hline & IL-6 (pg/L) & MMP-9 (ng/L) & TIMP-1 (ng/L) & MMP-9 / TIMP-1 ratio (\%) \\
\hline Control group 1 & $4.39 \pm 0.99$ & $1.68 \pm 0.46$ & $12.57 \pm 2.00$ & $13.77 \pm 4.37$ \\
FIRS group 1 & $42.68 \pm 6.98^{* *}$ & $48.48 \pm 10.2^{* *}$ & $106.07 \pm 6.61^{* *}$ & $45.95 \pm 10.96^{* *}$ \\
Control group 2 & $4.51 \pm 1.03$ & $4.80 \pm 1.51^{* *}$ & $60.93 \pm 7.37^{* *}$ & $7.92 \pm 2.45^{* *}$ \\
FIRS group 2 & $33.63 \pm 8.77^{\# \#}$ & $34.94 \pm 11.08^{\# \#}$ & $99.97 \pm 17.38^{\# \#}$ & $35.04 \pm 9.00^{\# \#}$ \\
\hline
\end{tabular}

FIRS, Fetal inflammatory response syndrome; IL-6, interleukin 6; MMP-9, matrix metallopeptidase 9; TIMP-1, tissue inhibitor matrix metalloproteinase 1 ; $* *$ indicates $p<0.01$ compared with Control group 1; ${ }^{\#}$ indicates $p<0.01$ compared with Control group 2.
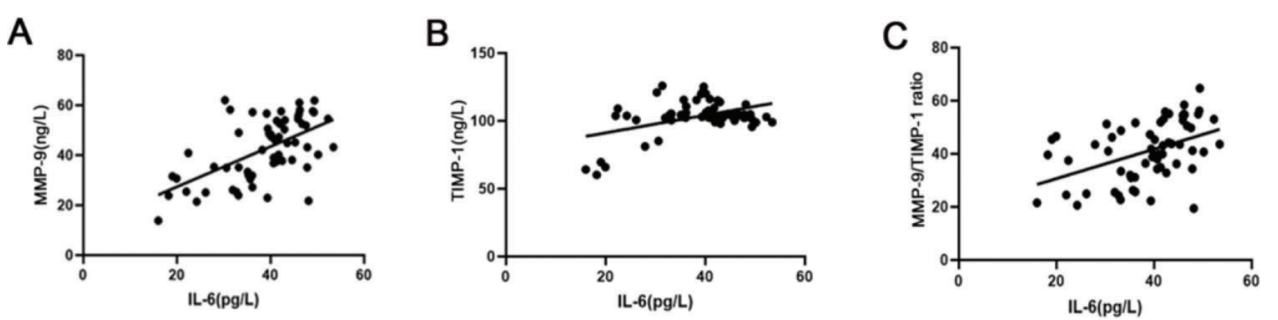

Figure 1 - The correlation between IL-6 and MMP-9, TIMP-1, and the MMP-9/TIMP-1 ratio in preterm infants. (A) Spearman's linear correlation results indicate that IL-6 was positively correlated with MMP-9. (B) Spearman's linear correlation results indicate that IL-6 was positively correlated with TIMP-1; (C) Spearman's linear correlation results indicate that IL- 6 was positively correlated with the MMP-9/TIMP-1 ratio. IL-6, interleukin 6; MMP-9, matrix metallopeptidase 9; TIMP-1, tissue inhibitor matrix metalloproteinase 1.

group $2(p>0.05)$. Additionally, the IL-6 level in Control Group 2 did not differ significantly from that in Control group 1 ( $p>0.05)$. However, the levels of MMP-9 and TIMP-1 in Control group 2 were significantly increased, whereas the MMP-9/TIMP-1 ratio was significantly decreased (all $p<0.01$ ) (Table 2).

The correlation between IL-6 and MMP-9, TIMP-1, and the MMP-9/TIMP-1 ratio

Based on Spearman's linear correlation analysis, IL-6 was positively correlated with the levels of MMP-9 and TIMP-1 and the MMP-9/TIMP-1 ratio ( $\mathrm{p}_{\text {MMP- } 9}<0.01, \mathrm{r}_{\text {MMP-9 }}=0.58$; $\mathrm{p}_{\text {TIMP- } 1}<0.01, \mathrm{r}_{\text {TIMP-1 }}=0.45 ; \mathrm{p}_{\text {MMP-9/TIMP-1 ratio }}<0.01, \mathrm{r}_{\text {MMP-9/ }}$ TIMP-1 ratio $=0.44$ ) (Figure $1 \mathrm{~A}-\mathrm{C}$ ).

The diagnostic value of cytokines in predicting FIRS

The critical values of MMP-9 levels, TIMP-1 levels, and the MMP-9/TIMP-1 ratio for predicting FIRS are shown in Table 3. The area under the curve (AUC) of MMP-9, TIMP-1, and the MMP-9/TIMP-1 ratio were 0.92, 0.90, and 0.95, respectively. The sensitivity and specificity of these indicators were above $90 \%$.

\section{Histological examination of placental tissues in preterm infants}

No pathological changes were observed in the placenta of subjects from the Control groups (Figure 2A). Chorioamnionitis was the most prevalent lesion observed in the chorion, and acute and chronic inflammatory cell infiltration was documented (Figure 2B). However, pathological changes in the placenta of subjects from the FIRS group showed inflammatory cell accumulation, bleeding, and necrosis. A large number of neutrophils were aggregated in the tissues showing acute inflammation, along with congestion and edema. Inflammatory cells such as neutrophils, lymphocytes, and monocytes were observed in the tissues showing chronic inflammation, with necrosis and proliferation (Figure 2C, 2D).

\section{Examination of amniotic fluid smears in preterm infants}

As shown in Figure 3B, 3C, the amniotic fluid smears revealed inflammatory cells, including a large number of neutrophils and few lymphocytes, aggregated in the samples from subjects in the FIRS group. However, the smear samples of subjects from the Control groups mainly showed exfoliated epithelial cells and minimal impurities (Figure 3A).

\section{Follow-up}

During follow-up, the incidence of BPD and myocardial injury was higher in FIRS group 1 than in Control group 1 $(p<0.05)$. Additionally, the incidence of myocardial injury was higher in FIRS group 2 than in Control group $2(p<0.05)$, whereas no significant difference was observed in the 
Table 3 - The diagnostic value of cytokines for predicting FIRS.

\begin{tabular}{lcccc}
\hline & Cutoff value & Sensitivity (\%) & Specificity (\%) & 95\% Cl \\
\hline MMP-9 & 21.61 & 0.98 & 0.91 & $0.86-0.98$ \\
TIMP-1 & 78.76 & 0.94 & 0.91 & 0.92 \\
MMP-9 / TIMP-1 ratio & 22.50 & 0.96 & 0.90 & $0.93-0.97$ \\
\hline
\end{tabular}

FIRS, Fetal inflammatory response syndrome; Cl, Confidence interval; AUC, Area under the curve; IL-6, interleukin 6; MMP-9, matrix metallopeptidase 9 ; TIMP-1, tissue inhibitor matrix metalloproteinase 1.

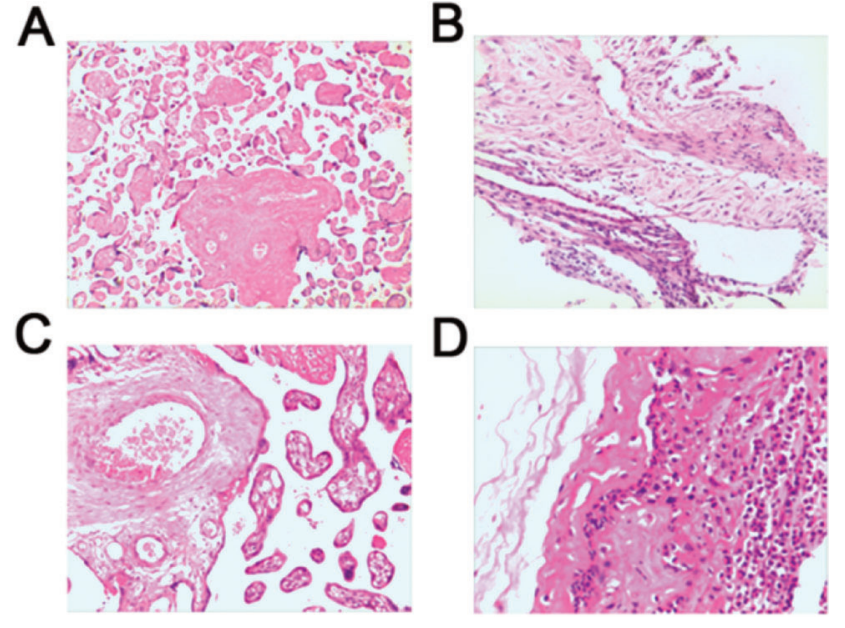

Figure 2 - Histological changes in placental tissues obtained from preterm infants were examined by HE staining. (A) Normal placental tissue from preterm infants demonstrates no obvious pathological change $(\times 200)$. (B) Chorionic membrane tissue demonstrates mild inflammatory cell infiltration $(\times 200)$. (C) Acute inflammatory placental tissues show congestion and edema, with aggregated neutrophils $(\times 200)$. (D) Chronic inflammatory placental tissues show necrosis, proliferation, neutrophils, lymphocytes, and monocytes $(\times 200)$. HE, hematoxylin and eosin.

incidence of BPD between the two groups $(p>0.05)$ (Tables 4 and 5).

Among preterm infants with FIRS, the incidence of BPD was higher in FIRS group 1 than in FIRS group $2(p<0.05)$, with no significant difference in the incidence of myocardial injury being observed between the two groups $(p>0.05)$. The incidence of BPD and myocardial injury did not significantly differ between Control group 1 and Control group $2(p>0.05)$ (Tables 4 and 5).

\section{DISCUSSION}

Preterm birth is classified as extremely preterm $(<28$ weeks), very preterm (28 to 32 weeks), and moderate to late preterm (32 to 37 weeks) based on the gestational age (15). It has been reported that preterm birth is closely related to FIRS (16). In the current study, the levels of IL-6, MMP-9, and TIMP-1, and the MMP-9/TIMP-1 ratio in the umbilical cord blood of infants presenting FIRS (gestational age of $\leqslant 32$ weeks and 32 to 37 weeks) were upregulated, and IL-6 levels were positively correlated with the MMP-9 and TIMP-1 levels and the MMP-9/TIMP-1 ratio. Additionally, these inflammatory indicators presented a certain diagnostic value for FIRS, which combined with the gestational age, were effective indicators for predicting the incidence of BPD and myocardial injury.
A previous review has summarized the association between the inflammatory response and premature birth, which indicates that IL-6 $(>11 \mathrm{pg} / \mathrm{mL})$ is considered an independent risk factor for the subsequent development of major neonatal morbidity (17). Furthermore, investigations regarding cytokines in the umbilical cord blood aid in the diagnosis of FIRS. For example, Rangel-Frausto et al. (18) have demonstrated that elevated IL- 6 levels precede the onset of preterm birth. A similar study has revealed that the inflammatory marker IL-6 is closely associated with tumor necrosis factor (TNF)- $\alpha$ in the umbilical cord blood at 22-34 weeks of gestation, and IL- 6 and TNF- $\alpha$ are of notable value for the diagnosis of FIRS (19). In our study, the levels of MMP-9 and TIMP-1 and the MMP-9/TIMP-1 ratio were higher in the FIRS groups (FIRS group 1 and FIRS group 2) than in the Control groups (Control group 1 and Control group 2), consistent with the reported IL-6 levels. Additionally, the levels of MMP-9 and TIMP-1 and the MMP-9/TIMP1 ratio revealed a good diagnostic value in predicting the incidence of FIRS. These results indicated that MMP-9, TIMP-1, and the MMP-9/TIMP-1 ratio may be predictive indicators for FIRS.

It is well known that inflammatory responses mediated via IL-6, IL-1 $\beta$, and TNF- $\alpha$ stimulate the secretion of MMPs $(20,21)$. MMPs include collagenases (MMP-1, MMP-8, and MMP-13), stromelysins (MMP-3, MMP-7, and MMP-10), and gelatinase (MMP-2 and MMP-9). MMP-9 is known as gelatinase- $\mathrm{B}$, which is a type 4 collagenase secreted by leukocytes and is related to fetal membrane damage (22). A previous study has reported that MMP-9 levels appear to be dependent on gestational age, with the highest levels observed at 33-36 weeks of gestation in premature infants with no BPD, and the TIMP-1 level was highest in full-term neonates (23). Likewise, Bednarek et al. (24) have reported that the MMP-9 level is dependent on gestational age in premature infants in the control group. Surprisingly, our study found that the levels of MMP-9 and TIMP-1 in Control group 2 were higher than those in Control group 1; however, the MMP-9/TIMP-1 ratio in Control group 2 was lower than that in Control group 1. This was attributed to the increased MMP-9 level, which was significantly lower than the TIMP-1 level in Control group 2. Similarly, the MMP-9/ TIMP-1 ratio was slightly lower in FIRS group 2 than in FIRS group 1. A previous study has demonstrated that the MMP9/TIMP-1 ratio is negatively correlated with gestational age, which is consistent with the findings of our study, indicating that lung development gradually matures with increasing gestational age $(14,25)$. Additionally, a previous study has indicated that the MMP-9/TIMP-1 ratio is closely correlated with the duration of oxygen supplementation, suggesting the extent of cardiopulmonary injury (14). In the current study, the incidence of BPD and myocardial injury was slightly lower in Control group 2 than in Control group 1; however, no significant differences were observed, which is consistent 

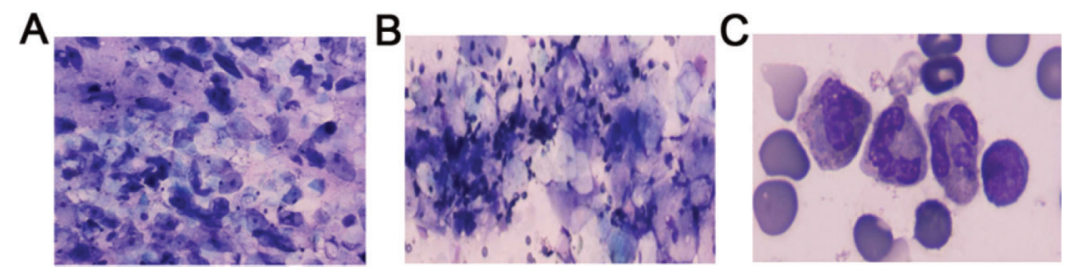

Figure 3 - Examination of amniotic fluid smears of preterm infants. (A) The amniotic fluid smear for normal preterm infants mainly shows exfoliated epithelial cells and a small number of impurities $(\times 400)$. (B) The amniotic fluid smear for preterm infants with FIRS shows inflammatory cells, mostly including neutrophils and a few aggregated lymphocytes $(\times 400)$. (C) Images of the amniotic fluid smear for preterm infants with FIRS were captured using oil immersion microscopy. FIRS, fetal inflammatory response syndrome.

Table 4 - The incidence of BPD in the different groups.

\begin{tabular}{lcccc}
\hline & Occurrence $(\mathbf{n})$ & Nonoccurrence $(\mathbf{n})$ & Total $(\mathbf{n})$ & Rate $(\%)$ \\
\hline Control group 1 & 2 & 23 & 25 & 3.00 \\
FIRS group 1 & 10 & 23 & 33 & $30.30^{*}$ \\
Control group 2 & 0 & 32 & 32 & 0 \\
FIRS group 2 & 1 & 27 & 28 & $3.57^{\& \&}$ \\
\hline
\end{tabular}

BPD, Bronchopulmonary dysplasia; FIRS, Fetal inflammatory response syndrome; *indicates $p<0.05$ compared with Control group 1; \&\&indicates $p<0.01$ compared with FIRS group 1.

Table 5 - The incidence of myocardial damage in the different groups.

\begin{tabular}{lcccc} 
& Occurrence $(\mathbf{n})$ & Nonoccurrence $(\mathbf{n})$ & Total $(\mathbf{n})$ & Rate $(\%)$ \\
\hline Control group 1 & 2 & 23 & 25 & 8.00 \\
FIRS group 1 & 11 & 22 & 33 & $32.33^{*}$ \\
Control group 2 & 1 & 31 & 32 & 3.13 \\
FIRS group 2 & 6 & 22 & 28 & $21.43^{\#}$ \\
\hline
\end{tabular}

FIRS, Fetal inflammatory response syndrome; *indicates $p<0.05$ compared with Control group 1; ${ }^{\text {indicates }} p<0.05$ compared with Control group 2.

with the trend of the MMP-9/TIMP-1 ratio. Furthermore, a high MMP-9/TIMP-1 ratio is considered a risk factor for lung damage (26). Therefore, the abovementioned reports suggest that the levels of MMP-9 and TIMP-1 and the MMP-9/TIMP1 ratio appeared to be dependent on gestational age in premature infants in Control groups.

In addition, the levels of MMP-9 and TIMP-1 are upregulated in premature infants with BPD and encephalopathy, which has been previously confirmed $(23,24)$. In the current study, the incidence of both BPD and myocardial injury were higher in FIRS group 1 than in Control group 1. However, the incidence of BPD did not significantly differ between FIRS group 2 and Control group 2. These results suggest that BPD may be closely correlated with gestational age. Our results were consistent with those reported by Mittendorf et al. (13), indicating that gestational age and low birth weight can act as major risks for BPD. Additionally, the current study revealed that the incidence of BPD in FIRS group 1 was higher than that in FIRS group 2. All obtained data suggested that the levels of MMP-9 and TIMP-1, combined with gestational age, may be novel indicators for predicting the incidence of BPD and myocardial injury.

Several reports have demonstrated that the MMP-9/TIMP1 ratio is associated with the development of some diseases in newborn infants, including bronchiectasis and lung infection $(27,28)$. In terms of BPD, Fukunaga et al. (14) have reported that in infants at a gestational age $<30$ weeks, the MMP-9/TIMP-1 ratio in the cord blood is related to the development of BPD. A subsequent study has reported that the MMP-9/TIMP-1 ratio plays an important role in the progression of BPD, and it was observed that Mn (III) tetrakis (4-benzoic acid) porphyrin chloride can effectively decrease the MMP-9/TIMP-1 ratio in a rat model (29). Therefore, the MMP-9/TIMP-1 ratio, combined with the gestational age, can be used to predict the possibility of BPD in premature infants with FIRS. Furthermore, we observed that the incidence of myocardial injury in the FIRS groups was higher than that in the Control groups. In the present study, the relationship between the MMP-9/TIMP-1 ratio and myocardial injury was in accordance with the findings of previous investigations reporting that the MMP-9/TIMP-1 ratio is related to brain injury (24). These results suggested that the MMP-9/TIMP-1 ratio was closely associated with the incidence of BPD (gestational age $\leqslant 32$ weeks) and myocardial injury (gestational age $<37$ weeks) in preterm infants.

\section{CONCLUSIONS}

In FIRS, the levels of IL-6, MMP-9, and TIMP-1, and the MMP-9/TIMP-1 ratio in the umbilical cord blood were upregulated, and IL-6 levels were positively correlated with the levels of MMP-9 and TIMP-1 and the MMP-9/TIMP-1 ratio. Furthermore, these inflammatory indicators presented a certain diagnostic value in FIRS; combined with gestational age, these markers were effective in predicting the incidence of BPD and myocardial injury. 


\section{ACKNOWLEDGMENTS}

This work was supported by the training of excellent clinical medical personnel and the basic research project and the Medical Science Research Key project of the Hebei province (20160163).

\section{AUTHOR CONTRIBUTIONS}

Jiang L conceived and designed this study; Zhang WH and Zhang HF provided materials and samples; Yan YW, Zhang WT, and Li M participated in data collection, analysis, and interpretation of the results. Yan YW wrote the manuscript. Shen Y, and Jiang L provided administrative support.

\section{REFERENCES}

1. Wen SW, Smith G, Yang Q, Walker M. Epidemiology of preterm birth and neonatal outcome. Semin Fetal Neonatal Med. 2004;9(6):429-35. https:// doi.org/10.1016/j.siny.2004.04.002

2. Martin JA, Hamilton BE, Osterman MJK. Births in the United States, 2018. NCHS Data Brief. 2019(346):1-8.

3. Pararas MV, Skevaki CL, Kafetzis DA. Preterm birth due to maternal infection: Causative pathogens and modes of prevention. Eur J Clin Microbiol Infect Dis. 2006;25(9):562-9. https://doi.org/10.1007/s10096-006-0190-3

4. Gomez R, Romero R, Ghezzi F, Yoon BH, Mazor M, Berry SM. The fetal inflammatory response syndrome. Am J Obstet Gynecol. 1998;179(1): 194-202. https: / /doi.org/10.1016/S0002-9378(98)70272-8

5. Chakraborti S, Mandal M, Das S, Mandal A, Chakraborti T. Regulation of matrix metalloproteinases: an overview. Mol Cell Biochem. 2003;253(1-2): 269-85. https://doi.org/10.1023/A:1026028303196

6. Lemaitre V, D'Armiento J. Matrix metalloproteinases in development and disease. Birth Defects Res C Embryo Today. 2006;78(1):1-10. https://doi. org $/ 10.1002 /$ bdrc. 20065

7. Prause O, Bozinovski S, Anderson GP, Lindén A. Increased matrix metalloproteinase- 9 concentration and activity after stimulation with interleukin-17 in mouse airways. Thorax. 2004 Apr;59(4):313-7. https:// doi.org/10.1136/thx.2003.008854

8. Robertson CM, Coopersmith CM. The systemic inflammatory response syndrome. Microbes Infect. 2006;8(5):1382-9. https://doi.org/10.1016/ j.micinf.2005.12.016

9. O'Reilly M, Sozo F, Harding R. Impact of preterm birth and bronchopulmonary dysplasia on the developing lung: long-term consequences for respiratory health. Clin Exp Pharmacol Physiol. 2013;40(11):765-73. https://doi.org/10.1111/1440-1681.12068

10. Alexander B, Kelly CE, Adamson C, Beare R, Zannino D, Chen J, et al. Changes in neonatal regional brain volume associated with preterm birth and perinatal factors. Neuroimage. 2019;185:654-63. https://doi.org/ 10.1016/j.neuroimage.2018.07.021

11. Jobe AH. The new bronchopulmonary dysplasia. Curr Opin Pediatr. 2011;23(2):167-72. https://doi.org/10.1097/MOP.0b013e3283423e6b

12. Zhong QH, Duan J, Liang K, Zeng J, Qi ZY, He XY. [A clinical study of growth and metabolism of small for gestational age infants]. Zhongguo Dang Dai Er Ke Za Zhi. 2019;21(5):458-62. https://doi.org/10.7499/ j.issn.1008-8830.2019.05.012

13. Mittendorf R, Covert R, Montag AG, elMasri W, Muraskas J, Lee KS, et al. Special relationships between fetal inflammatory response syndrome and bronchopulmonary dysplasia in neonates. J Perinat Med. 2005;33(5): 428-34. https://doi.org/10.1515/JPM.2005.076

14. Fukunaga S, Ichiyama T, Maeba S, Okuda M, Nakata M, Sugino N, et al. MMP-9 and TIMP-1 in the cord blood of premature infants developing
BPD. Pediatr Pulmonol. 2009;44(3):267-72. https://doi.org/10.1002/ppul. 20993

15. Platt MJ. Outcomes in preterm infants. Public health. 2014;128(5):399-403. https://doi.org/10.1016/j.puhe.2014.03.010

16. Bonadio J. Premature birth and the fetal inflammatory response syndrome. NeoReviews. 2016;17(5):e285-9. https://doi.org/10.1542/neo.17-5e285

17. Helmo FR, Alves EAR, Moreira RAA, Severino VO, Rocha LP, Monteiro MLGDR, et al. Intrauterine infection, immune system and premature birth. J Matern Fetal Neonatal Med. 2018;31(9):1227-33. https://doi.org/ 10.1080/14767058.2017.1311318

18. Rangel-Frausto MS, Pittet D, Costigan M, Hwang T, Davis CS, Wenzel RP. The natural history of the systemic inflammatory response syndrome (SIRS). A prospective study. Jama. 1995;273(2):117-23. https://doi.org/ 10.1001/jama.1995.03520260039030

19. Pilypienè I, Drazdienè N, Dumalakienè I, Vezbergienè N, Bartkevi ienè D, Silkunas M, et al. The significance of fetal inflammatory response syndrome in early and later adaptation of premature infants. Arch Gynecol Obstet. 2015 Jan;291(1):67-72.

20. Goldenberg RL, Andrews WW, Goepfert AR, Faye-Petersen O, Cliver SP, Carlo WA, et al. The Alabama Preterm Birth Study: umbilical cord blood Ureaplasma urealyticum and Mycoplasma hominis cultures in very preterm newborn infants. Am J Obstet Gynecol. 2008;198(1):43.e1-5. https:// doi.org/10.1016/j.ajog.2007.07.033

21. Sarandakou A, Giannaki G, Malamitsi-Puchner A, Rizos D, Hourdaki E, Protonotariou $\mathrm{E}$, et al. Inflammatory cytokines in newborn infants. Mediators Inflamm. 1998;7(5):309-12. https://doi.org/10.1080/096293 59890811

22. Chakrabarti S, Patel KD. Matrix metalloproteinase-2 (MMP-2) and MMP9 in pulmonary pathology. Exp Lung Res. 2005;31(6):599-621. https://doi. org /10.1080/019021490944232

23. Schulz CG, Sawicki G, Lemke RP, Roeten BM, Schulz R, Cheung PY. MMP-2 and MMP-9 and their tissue inhibitors in the plasma of preterm and term neonates. Pediatr Res. 2004;55(5):794-801. https://doi.org/ 10.1203/01.PDR.0000120683.68630.FB

24. Bednarek N, Svedin P, Garnotel R, Favrais G, Loron G, Schwendiman L, et al. Increased MMP-9 and TIMP-1 in mouse neonatal brain and plasma and in human neonatal plasma after hypoxia-ischemia: a potential marker of neonatal encephalopathy. Pediatr Res. 2012;71(1):63-70. https://doi. org $/ 10.1038 /$ pr.2011.3

25. Sweet DG, Curley AE, Chesshyre E, Pizzotti J, Wilbourn MS, Halliday HL, et al. The role of matrix metalloproteinases -9 and -2 in development of neonatal chronic lung disease. Acta Paediatr. 2004;93(6):791-6. https:// doi.org/10.1111/j.1651-2227.2004.tb03020.x

26. Ekekezie II, Thibeault DW, Simon SD, Norberg M, Merrill JD, Ballard RA, et al. Low levels of tissue inhibitors of metalloproteinases with a high matrix metalloproteinase-9/tissue inhibitor of metalloproteinase-1 ratio are present in tracheal aspirate fluids of infants who develop chronic lung disease. Pediatrics. 2004;113(6):1709-14. https://doi.org/10.1542/peds. 113.6.1709

27. Guan WJ, Gao YH, Xu G, Lin ZY, Tang Y, Gu YY, et al. Sputum matrix metalloproteinase- 8 and -9 and tissue inhibitor of metalloproteinase-1 in bronchiectasis: clinical correlates and prognostic implications. Respirology. 2015;20(7):1073-81. https://doi.org/10.1111/resp. 12582

28. Kong MY, Clancy JP, Peng N, Li Y, Szul TJ, Xu X, et al. Pulmonary matrix metalloproteinase-9 activity in mechanically ventilated children with respiratory syncytial virus. Eur Respir J. 2014;43(4):1086-96. https://doi. org/10.1183/09031936.00105613

29. Chang M, Bany-Mohammed F, Kenney MC, Beharry KD. Effects of a superoxide dismutase mimetic on biomarkers of lung angiogenesis and alveolarization during hyperoxia with intermittent hypoxia. Am J Transl Res. 2013;5(6):594-607. 